also to make cloud-chamber studies of the conditions preceding breakdown.

Grateful acknowledgment is made to the Council of the Durham Colleges for granting one of us (W.A.P.) a year's leave to pursue this work, and to the British Electrical and Allied Industries Research Association for financial support throughout the investigation.

W. A. Prowse

Electrical Engineering Dept.,

R. COOPER

Imperial College,

London, S.W.7.

Nov. 11.

' Cooper, R., J. Inst. Elect. Eng., 94, Pt. 3, 315 (Sept. 1947)

\section{Refraction Effects in Electron Diffraction and in Electron Microscopy}

ReCENTLy, Cowley and Rees ${ }^{1}$ reported some interesting work on the fine structure of electron diffraction patterns of crystals of magnesium oxide, cadmium oxide, etc. (multiple rings or spots) by application of high-resolution electron diffraction cameras. They confirmed the view of Sturkey and Frevel, who suggested that this fine structure arises from refraction at the faces of the regularly shaped crystal cubes. If the normals of the faces by which the beam of electrons enters and leaves the crystal lie in the same plane with the beam and the normal of the net plan $\Theta^{2}$, one can derive an expression relating the angular displacement of the beam $\varepsilon$ to the angles $\varphi_{1}$ and $\varphi_{2}$ (angle between face normal and incident and refracted electrons respectively) :

$$
\begin{aligned}
\varepsilon=\theta-\theta_{0}=\varepsilon_{1}+\varepsilon_{2}=V / 2 E\left( \pm \tan \varphi_{1} \pm \tan \varphi_{2}\right) & \\
\theta & <\varphi<\pi / 2-\theta
\end{aligned}
$$

( $V$ is inner potential, $E$ is accelerating potential, $\theta_{0}$ Bragg angle for $\left.V=0\right)$. This deviation having values of $10^{-4}$ radians $(V=10$ volts, $E=50,000$ volts, $\varphi=45^{\circ}$ ) or $3 \times 10^{-3} \mathrm{~cm}$. at a specimen-plate distance of $30 \mathrm{~cm}$., high resolution will be necessary to detect it.

This explanation is intimately connected with that given for the results obtained with electrolytically polished surfaces (Jacquet procedure) by electron diffraction and the electron microscope ${ }^{3}$. These surfaces are slightly waved so that the angle between the limiting face and the net planes $(\delta)$ has only a value of some degrees, whereas in the case considered above the faces are steeply inclined to the net planes. Therefore $\varepsilon$ becomes greater and the same refraction effect will be observed by normal resolution.

Thus $\varepsilon=\left(\tan \delta+\theta_{0}\right)\left[(+,) \sqrt{1-\frac{V}{E\left(\tan \delta+\theta_{0}\right)^{2}}}-1\right]$ $\left(\sin \theta \sim \theta, \sin \theta_{0} \sim \theta_{0}\right.$ ). Generally, one can put $V \mid E<\left(\tan \delta+\theta_{0}\right)^{2}$ also for electrolytically polished surfaces. Thus it follows

$$
\begin{array}{r}
\theta=\theta_{0}\left(1-V / 2 E \theta_{0}^{2}\left(1+\tan \delta / \theta_{0}\right)\right) \text { or } \\
\varepsilon=-V / 2 E\left(\tan \delta+\theta_{0}\right) .
\end{array}
$$

With $\delta \sim 5 \times 10^{-2}\left(3^{\circ}\right)$ and $\theta_{0} \sim 2 \times 10^{-2}$, one finds $\varepsilon \sim 15 \times 10^{-4}$, or half a millimetre at a platespecimen distance of $30 \mathrm{~cm}$., which can be seen without difficulty, $\tan \delta$ being always greater than 0 in the case of reflexion (there are always elevations transmitted), $\varepsilon$ will be less than 0 , or the reflected beam is displaced to smaller reflexion angles. If $\delta$ is continuously varying between 0 and $\delta_{\max }$, the patterns are enlarged to streaks as on electrolytically polished surfaces. For transmission patterns as referred to above, one has also $\tan \delta<0$ and therefore $\theta>\theta_{0}$.

Between the angles $\varphi, \theta$ and $\delta$ exists the relation $\delta=\pi / 2-(\varphi+\theta)$. To show the transition from eq. 2 to eq. 1, one has to put $\varphi>\theta$ as supposed for the derivation of (1), and one obtains

$$
\varepsilon=-(V / 2 E) \tan \varphi \text {. }
$$

The total deviation is given by the sum of $\varepsilon$ at entering and leaving the faces.

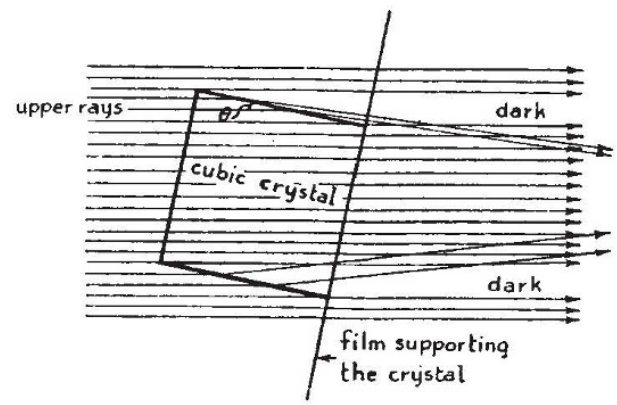

These considerations are also relevant to refraction effects in electron microscopy. A refraction effect may be of importance if well-shaped crystals, for example, cubic crystals, are transmitting electrons as shown in the diagram. The electrons being deflected by refraction out of their original direction, one would expect that the cube faces nearly parallel to the beam would appear as dark lines (a dark frame if the crystals are also somewhat tilted with their faces out of the plane of the paper). The refraction by the steeper faces is here neglected. Whether the refracted image is visible or not depends on the objective aperture and the inclination of the crystal. It seems to me that the dark bands which surround small inclined crystals of magnesium oxide ${ }^{5}$ may be explained in such a manner. The size of these bands is given by the crystal dimensions in the primary direction and the angle of inclination.

At very small angles $\left(\theta \leqslant \theta_{\lim }=\sqrt{V / E}=0.014\right.$ at $V=10$ volts and $E=50,000$ volts) the rays, in the figure the 'upper rays', are totally reflected, which gives as above a transmitted (dark) and a totally reflected (intense) image, the latter being more deflected $(2 \theta)$ than that normally refracted. Perhaps this effect would be found by a systematic study using crystals of greater dimensions, for which the bands are better resolved.

Laboratoires de l'O.N.E.R.A.,

\section{H. RAETHER}

Paris.

Sept. 16.

${ }^{1}$ Cowley and Rees, Proc. Phys. Soc., 59, 288 (1947). Nature, 158, 550 (1946). See also Sturkey and Frevel, Phys. Rev., 68, 56 (1945) and Hillier and Baker, Phys. Rev., 68, 98 (1945).

- If this condition is not fulflled one has to take a more general formuls derived by Cowley and Rees (ref. 1).

s Raether, H., Metaux et Corrosion (Jan. 1947).

' Kranert, W., Leise. K. H., and Raether, H., Z. Phys., 122, 248 (1944). In this paper the formula given for $\theta$ was $\theta=$ $\theta_{0} \sqrt{1-V / E \theta_{0}{ }^{2}\left(1+\tan \delta / \theta_{0}\right)}$, which had been derived by putting approximately $\theta+\theta_{0}=2 \theta_{0}$ : developed, it gives the exact

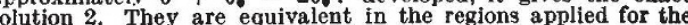
evaluation of electrolytically polished surfaces.

- For example, Heidenreich, R. D., and Sturkey, J. App. Phys. 16, 97 (1945), Fig. 1. Kinder, E., Naturwiss, 31, 149 (1943). This effect must not be confused with the parallel strips of at rongly inclined crystals the origin of which explains the dynamica theory, nor with the Fresnel diffraction strings in the out-of focus image of a crystal edge. 